\title{
Associação entre alergia ocular leve e ceratocone em crianças
}

Association between mild ocular allergy and keratoconus in children

Tatiana do Couto Morais Scaglioni ${ }^{1}$ (), Bruno Avelar Miranda ${ }^{2}$ (), Isadora Dutra Rodrigues ${ }^{1}$ (D), Pedro Paulo Leite dos Reis ${ }^{2}$ (])

Departamento de Córnea, Fundação Hilton Rocha, Belo Horizonte, MG, Brasil. 2 Programa de Pós-Graduação em Oftalmologia, Fundação Hilton Rocha, Belo Horizonte, MG, Brasil.

Scaglioni TC, Miranda BA, Rodrigues ID, Reis PP. Associação entre alergia ocular leve e ceratocone em crianças. Rev Bras Oftalmol. 2021;80(4)::e016.

doi:

Descritores:

Conjuntivite alérgica, Doenças da córnea; Ceratocone;

Oftalmologia; Segmento anterior do olho

Keywords:

Conjunctivitis, allergic; Corneal disease; Keratoconus; Ophthalmology; Anterior eye segment

Recebido: 19/01/2021

Aceito: 21/4/2021

Autor correspondente: Tatiana do Couto Morais Scaglioni Rua Coronel Luiz Pires, 80 - Centro CEP: 39400160 - Montes Claros-MG E-mail: tatiana@santacasaolhos.com.br

Instituição: Fundação Hilton Rocha, Belo Horizonte, MG, Brasil.

Fonte de auxílio à pesquisa: não financiado.

Conflitos de interesse: os autores declaram que não há conflitos de interesses.

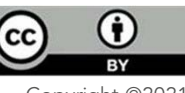

Copyright (C)2021

\section{RESUMO}

Objetivo: Avaliar a possibilidade de alterações precoces sugestivas de ectasia detectáveis no Pentacam em casos sutis de alergia ocular em crianças.

Métodos: Análise retrospectiva de 49 prontuários, com paciente de ambos os sexos e idades entre 3 e 14 anos. Do total de crianças estudadas, 31 apresentavam alergia ocular grau 1, e 18 não apresentavam qualquer sinal ou sintoma de alergia ocular, servindo como controles. Avaliaram-se diversos índices do Pentacam.

Resultados: Houve associação estatisticamente significativa nas variáveis "Df - variação do mapa da elevação anterior da córnea" e "Pentacam combinado", que podem estar relacionadas com a detecção precoce de ectasia corneana nesses pacientes.

Conclusão: Houve relação causal entre a prevalência da alergia ocular e alterações tomográficas na córnea, sugestivas de ceratocone em alguns pacientes provavelmente suscetíveis, podendo ser considerado um fator de risco para essa complicação.

\section{ABSTRACT}

Objective: To assess if early alterations detected by Pentacam in children suffering from mild ocular allergy are suggestive of ectasia.

Methods: A retrospective analysis of 49 medical records of patients of both sexes, aged between 3 and 14 years was performed. In this sample, 31 children suffered from grade I ocular allergy, and 18 presented no signs or symptoms of ocular allergy and comprised the control group. Several Pentacam indexes were evaluated.

Results: A statistically significant association was found in the variables "Df - variation of the anterior corneal elevation map" and "combined Pentacam", which can be related to the early detection of corneal ectasia in these patients.

Conclusion: There was a causal relation between prevalence of ocular allergy and tomographic alterations on the cornea, suggestive of keratoconus in some probably susceptible patients, which may be considered a risk factor for this complication. 


\section{INTRODUÇÃO}

A conjuntivite alérgica pode comprometer a qualidade de vida e afetar a visão. ${ }^{(1,2)}$ Sua prevalência parece estar aumentando em todo o mundo, tendo relação não apenas com a sensibilização a alérgenos, mas também com diferenças de etnia, clima, dieta, fatores socioeconômicos e exposição a diversos tipos de poluentes. ${ }^{(3)}$

De acordo com o Consenso Latino Americano de Alergia Ocular, essa patologia pode ser classificada baseada em sua severidade em graus de 1 a 4, levando-se em conta o tamanho das papilas conjuntivais, o grau de hiperemia, edema e cicatriz conjuntival e o acometimento corneano e limbar.(4) Essa doença acarreta diversas consequências e complicações oculares, e sua relação com o ceratocone já está bem estabelecida na literatura. (5,6)

O ceratocone é uma patologia ocular que aparece geralmente entre a segunda e terceira décadas de vida. ${ }^{(7)}$ É uma condição bilateral crônica caracterizada por afinamento corneano e astigmatismo irregular, levando frequentemente a prejuízo visual. ${ }^{(8)}$ Um dos fatores desencadeadores é o trauma mecânico da fricção ocular, secundário ao prurido, nos indivíduos geneticamente predispostos. ${ }^{\left({ }^{9}\right)}$ Nos casos de alergia ocular, é possível ser detectado precocemente por meio de topografia de córnea. ${ }^{(10)}$

Diferentemente do topógrafo, que só estuda a face anterior da córnea, o Pentacam, aparelho usado em nosso estudo, obtém informações da superfície anterior e posterior, empregando a técnica de imagem Scheimpflug. ${ }^{(11)}$ Apresenta índices topográficos e tomográficos da córnea com precisão e reprodutibilidade, possibilitando avaliar riscos de ectasia corneana. ${ }^{(2)}$ É sabido que formas prematuras de ceratocone costumam ter um curso mais agressivo. ${ }^{(13)}$

Assim, o objetivo deste estudo foi avaliar a possibilidade de alterações precoces sugestivas de ectasia detectáveis no Pentacam em casos sutis de alergia ocular em crianças

\section{MÉTODOS}

Trata-se de estudo retrospectivo em que foram analisados prontuários de 49 pacientes. Destes, 31 tinham diagnóstico de alergia ocular grau 1 de acordo com a classificação do Consenso Latino Americano de Alergia Ocular, sendo 22 do sexo masculino, com idades entre 3 a 14 anos, e 18 pacientes, sendo 12 meninas, com idades entre 6 e 12 anos, não tinham alterações oftalmológicas ou diagnóstico de alergia ocular e serviram como grupo controle.

As crianças foram atendidas no Ambulatório de Oftalmologia da Fundação Hilton Rocha, na cidade de Belo Horizonte, no período de outubro de 2013 a dezembro de 2015 e todas foram submetidas ao exame oftalmológico completo, além do exame de tomografia de córnea (Pentacam).

Devido à inexistência de banco de dados padronizados para crianças no Pentacam, os pacientes foram comparados com o grupo controle formado por crianças sem alterações oculares e na mesma faixa etária. Foram utilizados apenas os exames validados pelo aparelho. Foram excluídos do estudo pacientes com diagnóstico ou suspeita de ceratocone ou outra patologia que pudesse levar a alterações corneanas, casos de alergia ocular classificados de 2 a 4 de acordo com o Consenso Latino Americano de Alergia Ocular, incapacidade de realizar o exame e exames não validados pelo aparelho.

Avaliaram-se os seguintes índices do Pentacam: ART Max (Ambrósio's Relational Thickness); K Max (valor de ceratometria máxima); Df (variação do mapa da elevação anterior); Db (variação do mapa da elevação posterior); Dp (desvio do índice da progressão paquimétrica médio); Dt (variação da espessura mínima); Da (desvio do ART Max); $\mathrm{D}$ (valor da variação total). Consideramos também a possibilidade de o paciente ter pelo menos um dos índices analisados alterado, o que foi denominado neste estudo como "Pentacam combinado".

Utilizaram-se as funções de análise de dados e estatística descritiva para obtenção das médias, desvio-padrão, mínimas e máximas encontradas nos resultados de todos os exames realizados, assim como o percentual de exames alterados de cada grupo. A análise de correlação de Pearson foi utilizada, além do teste do qui-quadrado para análise de dados categóricos. Todos os resultados foram considerados significativos para uma probabilidade de significância inferior a 5\% $(\mathrm{p}<0,05)$, tendo, portanto, pelo menos 95\% de confiança nas conclusões apresentadas.

Este estudo foi postado na Plataforma Brasil (CAAE: 36424820.7.0000.5141), tendo sido aprovado pela Associação Educativa do Brasil/Faculdades Unidas do Norte de Minas, com número do parecer 4.272.378.

\section{RESULTADOS}

Foram analisados 98 olhos de 49 pacientes, de ambos os sexos, com idade variando de 3 a 14 anos (média de 9,1£2,3 anos). Do total de crianças estudadas, 31 apresentavam alergia ocular grau 1, e 18 não apresentavam qualquer sinal ou sintoma de alergia ocular.

Buscou-se avaliar alterações precoces de índices tomográficos do Pentacam daqueles pacientes com alergia ocular grau 1 em relação às crianças sem alergia ocular (Tabela 1). 


\section{REFERÊNCIAS}

1. Bielory L, Skoner DP, Blaiss MS, Leatherman B, Dykewicz MS, Smith N, et al. Ocular and nasal allergy symptom burden in America: the Allergies, Immunotherapy, and RhinoconjunctivitiS (AIRS) surveys. Allergy Asthma Proc. 2014;35(3):211-8.

2. Shaker M, Salcone E. An update on ocular allergy. Curr Opin Allergy Clin Immunol. 2016;16(5):505-10.

3. Miyazaki D, Fukagawa K, Okamoto S, Fukushima A, Uchio E, Ebihara $\mathrm{N}$, et al. Epidemiological aspects of allergic conjunctivitis. Allergol Int. 2020;69(4):487-95.

4. Santos MS, Alves MR, Freitas D, Sousa LB, Wainsztein R, Kandelman $\mathrm{S}$, et al. Ocular allergy Latin American consensus. Arq Bras Oftalmol. $2011 ; 74(6): 452-6$.

5. Naderan M, Shoar S, Rezagholizadeh F, Zolfaghari M, Naderan M Characteristics and associations of keratoconus patients. Cont Lens Anterior Eye. 2015;38(3):199-205.

6. Merdler I, Hassidim A, Sorkin N, Shapira S, Gronovich Y, Korach Z. Keratoconus and allergic diseases among Israeli adolescents between 2005 and 2013. Cornea. 2015;34(5):525-9.

7. Sorkin N, Varssano D. Corneal collagen crosslinking: a systematic review. Ophthalmologica. 2014;232(1):10-27.

8. Rabinowitz YS. Keratoconus. Surv Ophthalmol. 1998;42(4):297-319.

9. Dantas PE, Alves MR, Nishiwaki-Dantas MC. Topographic corneal changes in patients with vernal keratoconjunctivitis. Arq Bras Oftalmol. 2005;68(5):593-8.
10. Totan Y, Hepşen IF, Cekiç O, Gündüz A, Aydin E. Incidence of keratoconus in subjects with vernal keratoconjunctivitis: a videokeratographic study. Ophthalmology. 2001;108(4):824-7.

11. Chen D, Lam AK. Reliability and repeatability of the Pentacam on corneal curvatures. Clin Exp Optom. 2009;92(2):110-8.

12. Belim MW, Khachikian SS, Ambrósio Júnior R, Salomão M. Keratoconus/ Ectasia detection with the Oculus Pentacam: Belim/Ambrosio enhanced ectasia display. Highlights of Ophthalmology. 2007 [cited 2021 Apr. 21];35(6):5-12. Available from: https://www.oculus.de/uploads/media/belin.pdf

13. Léoni-Mesplié S, Mortemousque B, Touboul D, Malet F, Praud D, Mesplié N, et al. Scalability and severity of keratoconus in children. Am J Ophthalmol. 2012;154(1):56-62.e1.

14. Zheng Y, Huang G, Huang W, He M. Distribution of central and peripheral corneal thickness in Chinese children and adults: the Guangzhou twin eye study. Cornea. 2008;27(7):776-81.

15. Vieira MI, Germano AM, Zangalli C, Ferreira BG, Castro RS, Okanobo $A$, et al. Corneal evaluation in healthy Brazilian children using a scheimpflug topography system. J Clin Exp Ophthalmol. 2017 [cited 2021 Apr. 21];8(2):1-7. Available from: https://www.longdom.org/ open-access/corneal-evaluation-in-healthy-brazilian-children-using-ascheimpflugtopography-system-2155-9570-1000648.pdf

16. de Sanctis U, Aragno V, Dalmasso P, Brusasco L, Grignolo F. Diagnosis of subclinical keratoconus using posterior elevation measured with 2 different methods. Cornea. 2013;32(7):911-5.

17. Lema I, Sobrino T, Durán JA, Brea D, Díez-Feijoo E. Subclinical keratoconus and inflammatory molecules from tears. Br J Ophthalmol. 2009;93(6):820-4. 